\title{
Enhancing Closed-Loop Wireless Systems Through Efficient Feedback Reconstruction
}

\author{
Abdorreza Heidari, Student Member, IEEE, Farshad Lahouti, Member, IEEE, and Amir K. Khandani, Member, IEEE
}

\begin{abstract}
The closed-loop transmit diversity technique is used to improve the performance of the downlink channel in multiple-input multiple-output (MIMO) communication systems. The wideband code-division multiple-access standard endorsed by the Third Generation Partnership Project (3GPP) adopts a downlink closed-loop scheme based on partial channel state information (CSI) known as mode 1 . The information is fed back from the mobile unit to the base station through a low-rate uncoded feedback bit stream. In this paper, several reconstruction techniques are introduced to improve the performance of mode 1 of 3GPP in the presence of feedback error by taking advantage of the redundancy available in the bit stream of the CSI. We propose a number of algorithms for the reconstruction of beamforming weights at the base station with the constraint of a constant transmit power. The performance is examined within a simulated 3GPP framework. It is demonstrated that the proposed algorithms have substantial gain over the conventional method for low, moderate, and high mobile speeds. It is also shown that the proposed algorithms can substitute the antenna weight verification process done at the mobile unit in many cases. The proposed approach is applicable to other feedback schemes as well.
\end{abstract}

Index Terms-Antenna weight verification (AV), channel feedback, channel state information (CSI), closed-loop transmit diversity, downlink communication, frequency-division-duplex (FDD) wideband code-division multiple access (WCDMA), joint source-channel coding, mode 1 of the Third Generation Partnership Project (3GPP).

\section{INTRODUCTION}

$\mathbf{T}$ HE THIRD generation (3G) of mobile communications is intended to increase system performance, which is required to meet the rising demand for Internet and wireless services. In particular, the Third Generation Partnership Project (3GPP) [2] and 3GPP Two [3] have developed the wideband code-division multiple access (WCDMA) [4] and CDMA2000, respectively. Multiple antenna techniques, which are used in

Manuscript received September 2, 2005; revised August 22, 2006 and January 18, 2007. This work was supported in part by Bell Mobility, Communications and Information Technology Ontario (CITO), and by the Natural Sciences and Engineering Research Council of Canada (NSERC). This paper was presented in part at the IEEE Vehicular Technology Conference, Dallas, TX, September 2005. The review of this paper was coordinated by Prof. J. Shea.

A. Heidari and A. K. Khandani are with the Coding and Signal Transmission Laboratory, Department of Electrical and Computer Engineering, University of Waterloo, Waterloo, ON N2L 3G1, Canada (e-mail: reza@ cst.uwaterloo.ca; khandani@cst.uwaterloo.ca).

F. Lahouti is with the School of Electrical and Computer Engineering, College of Engineering, University of Tehran, Tehran 14174, Iran (e-mail: lahouti@ieee.org).

Color versions of one or more of the figures in this paper are available online at http://ieeexplore.ieee.org.

Digital Object Identifier 10.1109/TVT.2007.900506
3G systems, are known to enhance the capacity and quality of wireless communication [5] by exploiting the available spatial diversity. On the other hand, the improvement of the downlink capacity is one of the main challenges of the 3G systems because many of the proposed services are expected to be downlink intensive. Therefore, transmit antenna diversity has been considered, which is typically suitable for downlink applications [6].

Transmit diversity can be used with or without channel state information (CSI) at the transmitter, which is called closed loop or open loop, respectively. In the $3 \mathrm{G}$ evolution, openloop techniques such as Orthogonal Transmit Diversity [6] and Space-Time Transmit Diversity [7], and closed-loop techniques such as Switched Transmit Diversity and beamforming (also called Transmit Adaptive Array) [6], have been considered. 3GPP has included Alamouti Space-Time Coding [8] and one beamforming mode as part of the WCDMA frequency-divisionduplex (FDD) downlink system [9]. Generally speaking, openloop schemes are more robust as they do not need any CSI, whereas closed-loop schemes usually provide higher capacities. Closed-loop schemes are known to be effective for low-speed mobile users but fail at high mobile speeds [6].

A beamforming scheme enables the transmit array to beamform the transmit signal according to a particular channel state. In a multiple-input multiple-output (MIMO) system, when there is adequate CSI available at the transmitter, beamforming strategy is optimal in terms of system capacity [10]. With beamforming, the transmissions from different antenna elements at the base station constructively add at the receiver, which improves the received signal-to-noise ratio (SNR). However, this improvement requires that the transmitter has a fairly accurate knowledge of the parameters of the channel to the intended receiver. This is difficult to achieve as these parameters are time varying. Furthermore, in a practical communication system, feedback data are subject to imperfections such as quantization noise [11], feedback error [11], [12], and feedback delay [13]. Maintaining the closed-loop performance by using the available imperfect feedback data is an important issue for practical systems.

This paper presents methods to mitigate the problems of the closed-loop mode 1 of $3 \mathrm{GPP}$ in the presence of feedback error and to improve the performance of the underlying weight reconstruction algorithm. There are few works that address the problems of the closed-loop mode of the 3GPP standard, e.g., [14]. Here, we propose an approach to enhance the performance of mode 1 of $3 \mathrm{GPP}$ at different mobile speeds. Similarly, our approach is applicable to other closed-loop schemes. It is shown that the proposed algorithms result in substantial savings in 


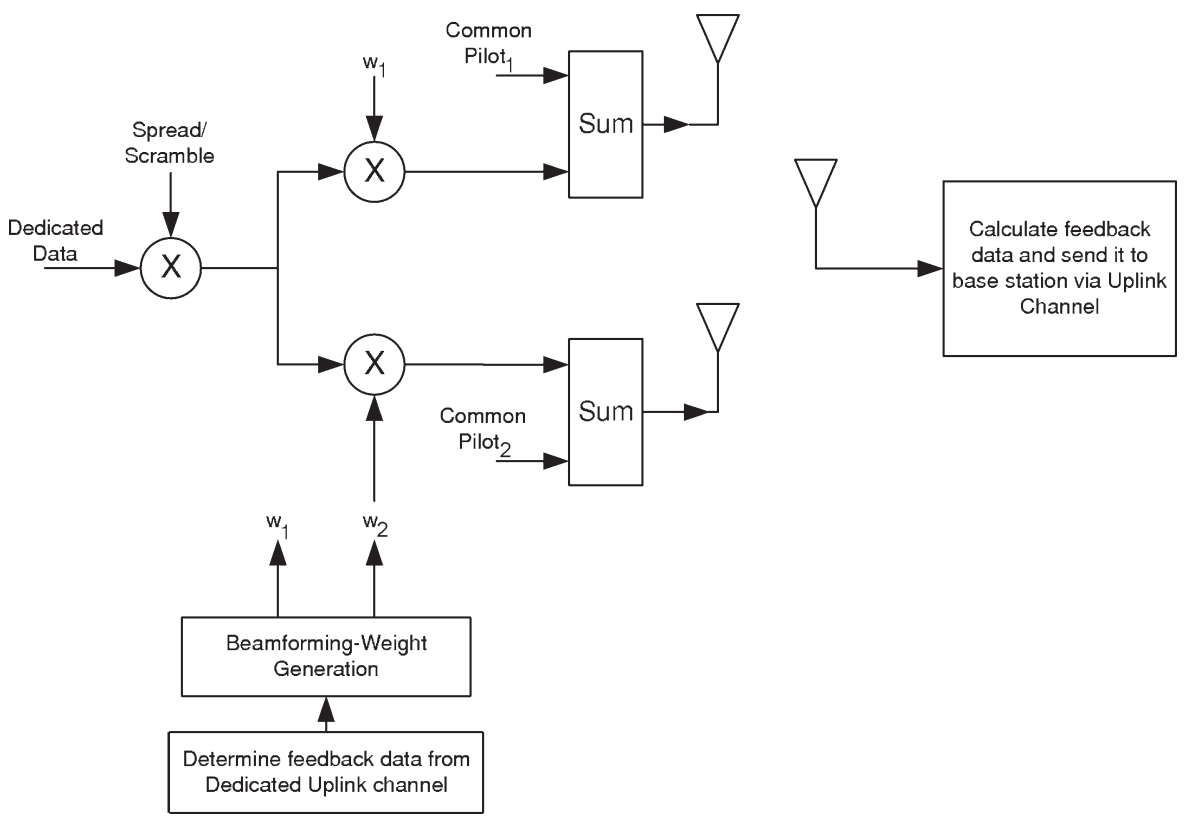

Fig. 1. Closed-loop modes of a 3GPP system.

base-station transmit power in comparison with the suggested reconstruction scheme for mode 1 in the 3GPP standard. The algorithms are compatible with the current standard framework, and they are appropriate for practical mobile systems in terms of robustness and complexity. Throughout this paper, our assumptions and simulation parameters are consistent with the WCDMA FDD closed-loop mode.

The structure of the rest of this paper is as follows. Section I-A includes a general description of a closed-loop system as well as our notations and channel model. In Section I-B, some conventional channel feedback schemes are examined, and Section I-C introduces our feedback model. Section II describes the algorithm of mode 1 of the 3GPP standard and its problems and challenges in the presence of feedback error. Section III explains our approach to reconstruct the beamforming weights and elaborates on the proposed methods and algorithms. Finally, Section IV shows the simulation results and compares the performance of our proposed algorithms with the conventional methods in various conditions.

\section{A. Closed-Loop Systems}

Fig. 1 shows a functional diagram of the closed-loop mode of the downlink 3GPP standard [9]. A general description of the feedback system follows. After the channel is estimated at the receiver by using the transmitted pilots, the CSI is quantized and sent back to the transmitter. The transmitter computes the required beamforming coefficients and applies them to the transmit antennas. In practice, the feedback channel has a low rate. Furthermore, there are delay and potential errors in the feedback channel. In spite of these imperfections in the feedback data, the closed-loop schemes perform significantly better than the open-loop schemes at low mobile speeds [6].

Consider a system with $M$ transmit antennas and one receive antenna. A flat-fading channel is considered from each transmit antenna to the receive antenna. Dropping the time indices for simplicity, we write

$$
r=h^{T} x+\eta
$$

where $r$ is the received signal at the receiver

$$
h=\left[h^{(1)}, \ldots, h^{(M)}\right]^{T} \in \mathbb{C}^{M}
$$

is the channel coefficient complex vector, where $h^{(m)}$ represents the channel between the $m$ th transmit antenna and the receive antenna, and

$$
x=\left[x^{(1)} \cdots x^{(M)}\right]^{T} \in \mathbb{C}^{M}
$$

represents the channel input vector. $\eta$ is a complex circularly symmetric additive white Gaussian noise (AWGN) with variance $N_{0}, \eta \sim \mathcal{N}\left(0, N_{0}\right)$. There is a constraint on the total transmit power $E\left[\|x\|^{2}\right]=\sum_{m=1}^{M} E\left[\left|x^{(m)}\right|^{2}\right] \leq M$.

For the channel model, we consider a Rayleigh fading model, and so, $h^{(m)}$ 's, $m=1, \ldots, M$, are zero-mean independent identically distributed circularly symmetric Gaussian random variables. Each coefficient is expressed as

$$
h^{(m)}=\alpha^{(m)} e^{j \phi^{(m)}}
$$

where $\alpha^{(m)}$ and $\phi^{(m)}$ are the amplitude and phase, respectively. It is well known that $\alpha^{(m)}$ has a Rayleigh distribution [15]

$$
p\left(\alpha^{(m)}=a\right)=a e^{-a^{2} / 2}, \quad a \geq 0
$$

and $\phi^{(m)}$ has a uniform distribution

$$
p\left(\phi^{(m)}=\theta\right)=\frac{1}{2 \pi}, \quad 0 \leq \theta<2 \pi .
$$


It is also known that the autocorrelation function of the fading for the 2-D isotropic scattering and an omnidirectional receiving antenna is given by [16]

$$
R(t, t-\delta)=\frac{E\left[h(t) h^{*}(t-\delta)\right]}{\sigma_{h}^{2}}=J_{0}\left(2 \pi f_{d} \delta\right)
$$

where $\sigma_{h}^{2}$ is the fading power, $J_{0}(\cdot)$ is the first-kind Bessel function of the zero order, $f_{d}$ is the Doppler frequency, and $\delta$ is the time difference.

Jakes' fading generator [17], which is a popular simulator for mobile fading channels, has been proposed based on the properties given in (5)-(7). It utilizes a number of low-frequency oscillators to generate a fading signal for a given mobile speed. The original Jakes' model generates a nonstationary signal. For the simulations in this paper, we use a modified Jakes' fading generator suggested in [16] to generate a stationary signal.

In our analysis, we assume that all the elements of the channel vector $h$ are known at the receiver. This assumption allows for a better comparison between different schemes in the presence of feedback imperfection regardless of the channel estimation errors. It is noteworthy that in the 3GPP systems, there is a separate common pilot channel with a relatively large proportion of the base station power, which allows each receiver to accurately estimate its channel coefficients [18], and this supports the above assumption.

\section{B. Beamforming}

In a closed-loop system, channel input $x$ should be appropriately selected according to the channel state [19]. Controlling the channel input can be accomplished with a conventional beamformer that applies some weights to the transmitted signal at each antenna. A beamforming scheme can be expressed as $x=w s$, where $w=\left[w^{(1)} \cdots w^{(M)}\right]^{T} \in \mathbb{C}^{M}$ is the antenna weight vector, and $s$ is the transmitted symbol. Without losing generality, we assume that $\|w\|^{2}=1$, which signifies that the beamformer does not change the total transmit power.

The received signal $r$ is a complex number that is the superposition of the signals from different channels, as well as the noise, i.e.,

$$
r=h^{T} w s+\eta=\left(\sum_{m=1}^{M} h^{(m)} w^{(m)}\right) s+\eta .
$$

In the decoding process at the receiver, the combining weight $v \in \mathbb{C}$ is applied as [15]

$$
z=v^{H} r=v^{H} h^{T} w s+v^{H} \eta
$$

where $z$ is used to calculate the output $\log$-likelihood ratio values (refer to [20] for further information on different combining schemes in WCDMA). It has been shown [21] that to minimize the average probability of error in a MIMO system (and therefore in a multiple-input single-output system like ours), $w$ and $v$ should be jointly selected to maximize the instantaneous SNR. Noting (9), we have

$$
\mathrm{SNR}_{\mathrm{inst}}=\left|h^{T} w\right|^{2} \frac{E_{s}}{N_{0}}
$$

where $E_{s}=E\left[|s|^{2}\right]$. It is observed that the SNR does not depend on $v$ in the case that there is one receive antenna (the case considered in the current work). As will be shown later, the accuracy of $v$ plays an important role in the overall system performance.

There are various methods to select the antenna weights to be applied at the transmitter. A number of conventional schemes to select $\mathrm{w}$ are given next.

1) Ideal Feedback: Maximizing the instantaneous SNR in (10), the optimum weights are found as

$$
w^{\text {ideal }}=\arg \max _{w}\left|h^{T} w\right|^{2}
$$

subject to the constraint $\|w\|^{2}=1$, which results in [22] $w^{\text {ideal }}=h^{*} /\|h\|$.

2) Cophase Feedback: The ideal feedback requires phase and amplitude information of the beamforming vector. A cophase feedback scheme corrects the phases of the signals received from different channels and adds them coherently without using the amplitude information of the channel. The cophase feedback algorithm may be shown as $w^{(1)}=1 / \sqrt{M}$ and $w^{(m)}=1 / \sqrt{M} e^{-j\left(\phi^{(m)}-\phi^{(1)}\right)}$ for $m=2, \ldots, M$.

It has been shown that the ideal feedback and cophase feedback schemes enhance the (average) received SNR by a factor of $M$ and $1+(M-1) \pi / 4$, respectively, with respect to the transmit $\mathrm{SNR}\left(\mathrm{SNR}_{\mathrm{tx}}=E_{s} / N_{0}\right)$ [13], [22]. This SNR gain is used as a figure of merit in an uncoded system. For $M=2$, the SNR gains are 3.0 and $2.5 \mathrm{~dB}$, respectively. Therefore, by using the phase-only information, we are losing only $0.5 \mathrm{~dB}$ of SNR (and at maximum $1.0 \mathrm{~dB}$ for a large number of transmit antennas). This confirms that the channel phase information is usually more important than the amplitude information.

3) Quantized Feedback: In practice, a limited capacity is available in the feedback channel. Therefore, for the quantization of the CSI, an efficient source-coding scheme should be used. There are a variety of works on quantization of feedback data, e.g., [19], [23]-[26].

The WCDMA standard has a closed-loop mode and suggests a reconstruction scheme for it: Mode 1, which is adopted from the cophase feedback scheme, is described in the next section. Mode 1 is initially designed to provide service for low to moderate mobile speeds [27]. However, its performance degrades at higher mobile speeds. Improving the performance at higher mobile speeds is of great importance as it helps to accommodate a larger number of mobile users. This is one of the objectives of this paper, which is achieved by using the feedback data more efficiently in reconstructing the underlying beamforming vector.

\section{Our Feedback Model}

In this paper, we focus on closed-loop systems with (quantized) cophase feedback. It is assumed that $M=2$; hence, 


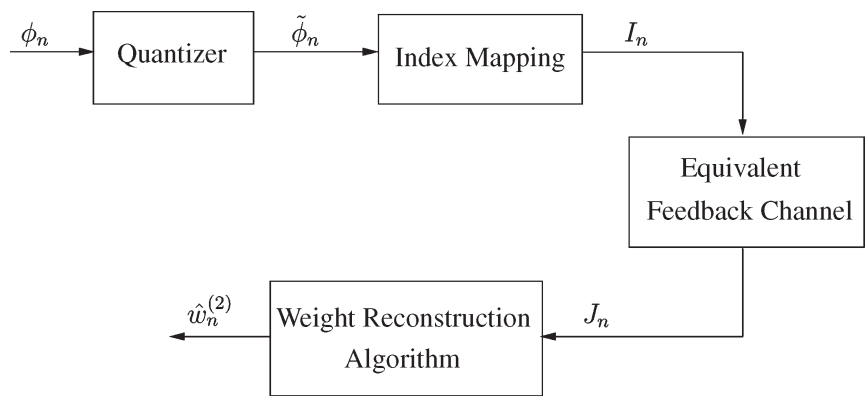

Fig. 2. Block diagram of the feedback link.

$h_{n}=\left[h_{n}^{(1)}, h_{n}^{(2)}\right]$, and $w_{n}=\left[w_{n}^{(1)}, w_{n}^{(2)}\right]$, where $n$ is the time index. It is also assumed that the first beamforming weight is constant, i.e., $w_{n}^{(1)}=1 / \sqrt{2}$, and $w_{n}^{(2)}$ is constructed from the feedback data. $\phi_{n}=\angle h_{n}^{(2)}-\angle h_{n}^{(1)}$ is the cophase that is quantized and fed back from the mobile to the base station. As it is assumed that beamforming does not change the transmit power, hence $\left|w_{n}^{(2)}\right|=1 / \sqrt{2}$.

Fig. 2 shows the block diagram of the feedback link, including the quantization at the receiver and the reconstruction at the transmitter. $\tilde{\phi}_{n}$ is the quantized cophase, and $I_{n}$ is the respective index. $I_{n}$ is sent through the equivalent feedback channel, and $J_{n}$ is received at the base station ( $I_{n}$ and $J_{n}$ are also called feedback symbols). $J_{n}$ could be a hard-decided symbol or a soft output of the channel. For an error-free feedback channel, $J_{n}=I_{n}$. Here, a memoryless feedback channel is assumed, which makes our analysis easier. This assumption is valid in practice as the feedback has a low symbol rate.

\section{Closed-Loop Mode 1 of 3GPP}

The closed-loop mode 1 of the 3GPP standard [9] is an example of the model introduced in Section I-C. In mode 1, the quantization of the phase $\phi_{n}$ is subject to a special framing structure, as shown in Fig. 3. Each (uplink) frame has a duration of $10 \mathrm{~ms}$ and includes 15 slots. Each slot contains a number of data symbols depending on the data rate. For each slot, 1 bit of feedback data is sent from the mobile unit, which results in a feedback stream of $1500 \mathrm{~b} / \mathrm{s}$. The feedback bit is determined by one of the two 1-bit quantizers $Q_{0}$ and $Q_{1}$, according to Fig. 3, where $Q_{0}=\{0, \pi\}$, and $Q_{1}=\{\pi / 2,-\pi / 2\}$. Showing the relative place of each slot in a frame, we use a framed time index $\tau=n \bmod 15$; hence, in a frame, $\tau=0,1, \ldots, 14$.

At the base station, the received feedback data bits are dequantized to $\hat{\phi}_{n}$ phase stream according to Fig. 3, i.e., $\hat{\phi}_{n} \in$ $Q_{\tau \bmod 2}$. The weight $w_{n}^{(2)}$ is constructed from the two recent phases, i.e., one from a $Q_{0}$ slot and one from a $Q_{1}$ slot. The reconstruction scheme can be shown as

$$
\hat{w}_{n}^{(2)}= \begin{cases}\frac{1}{2}\left(e^{j \hat{\phi}_{n}}+e^{j \hat{\phi}_{n-1}}\right), & \tau \neq 0 \\ \frac{1}{2}\left(e^{j \hat{\phi}_{n}}+e^{j \hat{\phi}_{n-2}}\right), & \tau=0 .\end{cases}
$$

Note that at each time, (12) selects $\hat{w}_{n}^{(2)}$ from a set of four predefined weights. This construction also guarantees that $\left|\hat{w}_{n}^{(2)}\right|=$ $1 / \sqrt{2}$ for all $n$.
It is observed in Fig. 3 that $\tilde{\phi}_{n}$ (and similarly $\hat{\phi}_{n}$ ) can attain one of four values, i.e., $\tilde{\phi}_{n} \in\{-\pi / 2,0, \pi / 2, \pi\}$. Therefore, according to Fig. 2, the index $I_{n}$ (and similarly $J_{n}$ ) could be defined as $I_{n} \in\{0,1,2,3\}$, which corresponds to $\tilde{\phi}_{n} \in$ $\{-\pi / 2,0, \pi / 2, \pi\}$, respectively. It is notable that these 2-bit symbols are constructed from the 1-bit feedback and the time information $\tau$. Furthermore, (12) shows that the weight reconstruction block of mode 1 can be demonstrated as in Fig. 4. Note that the weight reconstruction algorithm has been separated into two parts and is potentially suboptimum.

\section{A. Effect of Feedback Error on Performance}

One of the major problems associated with downlink beamforming is the presence of errors in the (uncoded) feedback stream. If a feedback command for the base station becomes corrupted during the transmission in the uplink slot, an incorrect antenna weight vector will be applied at the transmitter. There are two consequences for such an error. First, the received signal power is smaller because a nonoptimum weight is applied. However, simulations show that the performance degradation due to this effect is rather small.

The second consequence is much more serious. Assume that $\hat{w}$ is applied at the transmitter. Similar to (9), we have

$$
z=v^{H} h^{T} \hat{w} s+v^{H} \eta
$$

where $z$ is the decoding metric. Hence, the receiver should be able to calculate $h^{T} \hat{w}$ (called dedicated channel estimate [28]) for the calculation of the optimum $v$. The mobile unit obtains the dedicated channel estimate by combining the estimates for the individual antennas from the common pilots with the assumed weight vector $w$. However, each time a feedback error occurs, $w$ at the mobile unit is different from the actual $\hat{w}$ that is applied at the base station, because the mobile unit is unaware of the error occurrence in the feedback channel. Each of these mismatches in the dedicated channel estimate can potentially result in an error in the decoding process. Therefore, an error floor proportional to the feedback error is imposed on the performance.

The loss of the closed-loop gain because of the feedback error (the first effect) has been analyzed in [11]. To minimize the effect of the mismatch problem (the second effect), a technique called antenna weight verification (AV) [28] has been suggested. The AV technique is explained in the next section.

\section{B. Antenna Weight Verification}

In an ideal $\mathrm{AV}$, the mobile unit always knows the exact weight $\hat{w}$ applied at the base station. An ideal AV theoretically eliminates the mismatch effect, but it is practically impossible to implement when the feedback information is corrupted. In a practical AV algorithm, the mobile station considers the possibility of an erroneous feedback transmission. This is done by a hypothetical comparison between the common-pilot-based channel estimate and the one obtained from a few training symbols in the dedicated channel, which include the effect of $\hat{w}$. An AV algorithm for mode 1 of $3 \mathrm{GPP}$ has been suggested in 


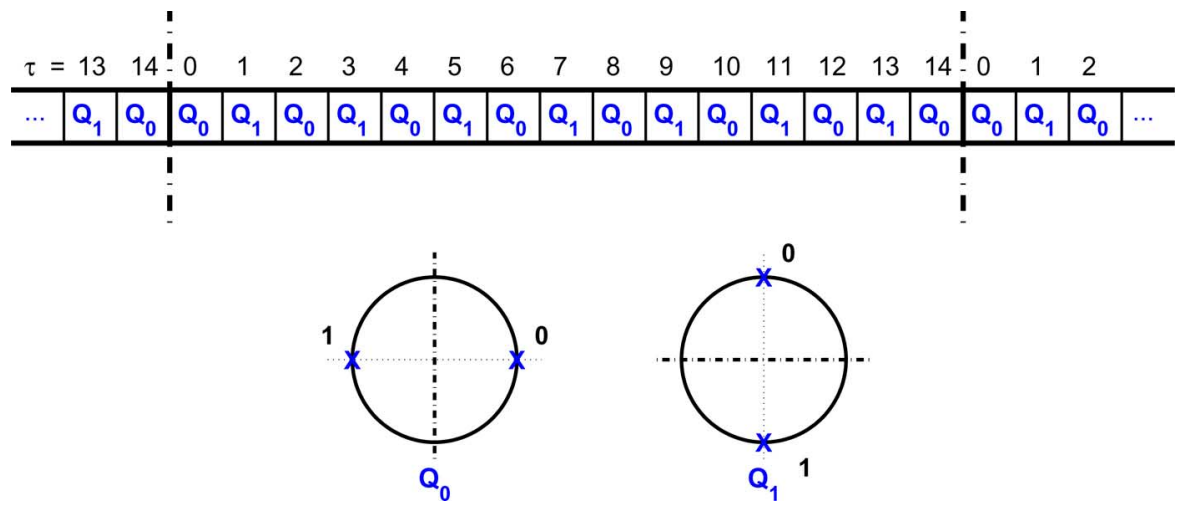

Fig. 3. Framing structure and the quantizers of mode 1 of 3 GPP.

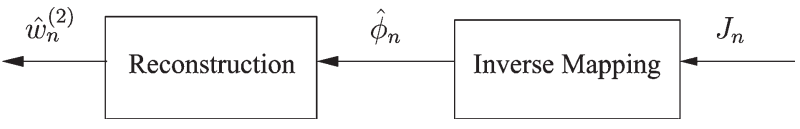

Fig. 4. Block diagram of the weight reconstruction for mode 1 of $3 \mathrm{GPP}$.

the annex to [9], and the performance of the AV algorithm has been analyzed in [29]. A trellis-based AV algorithm has been proposed in [18] to improve the performance of the verification process.

However, the AV technique has some drawbacks. Applying an $\mathrm{AV}$ algorithm requires extra calculations at the mobile unit and special dedicated preamble bits to be transmitted to all the users. Also, the structure of an AV algorithm is based on the beamforming scheme applied at the transmitter, which means that the $\mathrm{AV}$ algorithm is different for each beamforming algorithm. Furthermore, AV cannot decrease the loss of the closed-loop gain (first effect), as it is implemented at the mobile unit. Due to these problems, there is a tendency to find an alternative technique for AV (for example, see [30]).

In the next section, we introduce a new approach to improve the performance of the beamforming scheme, especially in the presence of feedback error. In this approach, the redundancies available in the feedback data are exploited to find an improved estimate of the antenna weight without the need for any preamble data sequence. Our approach helps solve both of the aforementioned problems as it provides a better reconstruction scheme for the weights. It decreases the effect of the feedback error that reduces the mismatch effect. It is notable that our algorithms can be used along with an AV algorithm. However, the performance results show the possibility of using our algorithms without the need for an AV algorithm in some cases. Comparison between the conventional method in conjunction with the Ideal AV and one of our algorithms shows that at low to moderate mobile speeds, our algorithm achieves the same performance without using AV.

\section{EFFICIENT RECONSTRUCTION OF BEAMFORMING WEIGHTS}

In the sequel, a feedback link consistent with Fig. 2 and defined in Section I-C is assumed. The framing structure and the quantization scheme of mode 1 of the 3GPP standard (described in Section II) is preserved here. However, more efficient
TABLE I

CODEBOoK Size

\begin{tabular}{|c|c|c|c|c|}
\hline$\mu$ & 1 & 2 & 3 & 4 \\
\hline \hline $4^{\mu}$ & 4 & 16 & 64 & 256 \\
\hline Codebook Size & 4 & 12 & 32 & 80 \\
\hline
\end{tabular}

reconstruction algorithms are presented. Unlike the method suggested in the standard, the effect of feedback error is considered in the reconstruction algorithms. Feedback delay is not considered here and could be the subject of a future research. The next section elaborates on a minimum mean-squared error (MMSE) criteria for the reconstruction that results in a number of proposed algorithms. Also, using a maximum a posteriori probability (MAP) criteria, other algorithms are presented.

\section{A. MMSE Approach}

The fundamental theorem of estimation states that given the received sequence $\underline{J}_{n}=\left[J_{1}, J_{2}, \cdots, J_{n-1}, J_{n}\right]$, the MMSE estimate of the weight is $\check{w}_{n}^{(2)}=E\left[w_{n}^{(2)} \mid \underline{J}_{n}\right]$ ( $\breve{w}$ is read omega(heck). It has been shown in [31] that the above formula can be approximated by

$$
\check{w}_{n}^{(2)}=\sum E\left[w_{n}^{(2)} \mid \underline{I}_{n}^{n-\mu+1}\right] P\left(\underline{I}_{n}^{n-\mu+1} \mid \underline{J}_{n}\right)
$$

where the summation is over all the possible $\mu$-fold sequences of $\underline{I}_{n}^{n-\mu+1}=\left[I_{n-\mu+1}, \cdots, I_{n-1}, I_{n}\right]$. In the MMSE sense, the formula is asymptotically optimum for sufficiently large values of $\mu$ [31].

In (14), $E\left[w_{n}^{(2)} \mid \underline{I}_{n}^{n-\mu+1}\right]$ is a nonlinear estimator for the weight. In fact, it defines a codebook as

$$
w_{\mathrm{CB}}\left(\underline{i}_{n}^{n-\mu+1}\right)=E\left[w_{n}^{(2)} \mid \underline{I}_{n}^{n-\mu+1}=\underline{i}_{n}^{n-\mu+1}\right]
$$

where $\underline{i}_{n}$ is a possible realization of $\underline{I}_{n}$. It is shown in Appendix B that the codebook has $(\mu+1) 2^{\mu}$ codewords, which is less than $4^{\mu}$ possible codewords. This is because there are some constraints on the value of $i_{n}$ imposed by the framing structure discussed earlier. Table I shows the codebook size for some typical values of $\mu$.

1) Trellis Structure: For capturing the redundancies in the feedback bit stream, we assume that the bit stream follows a Markov model of order $\gamma$. A trellis structure is set up based on the Markov model to exploit the redundancies. The states 
TABLE II

REDUNDANCIES $R(\gamma)$ FOR DIFFERENT MEMORY DEPTHS $\gamma$ AND DIFFERENT MOBILE SPEEDS

\begin{tabular}{|c|c|c|c|c|c|}
\hline$\gamma$ & 0 & 1 & 2 & 3 & 4 \\
\hline \hline$v=1$ & 0.01 & 0.78 & 1.61 & 1.67 & 1.69 \\
\hline$v=5$ & 0.01 & 0.78 & 1.48 & 1.53 & 1.55 \\
\hline$v=25$ & 0.01 & 0.76 & 1.12 & 1.14 & 1.16 \\
\hline$v=100$ & 0.01 & 0.73 & 0.75 & 0.76 & 0.78 \\
\hline
\end{tabular}

of the trellis are defined as $S_{n}=\underline{I}_{n}^{n-\gamma+1}$. There are in total $(\gamma+1) 2^{\gamma}$ possible states (see Appendix B). Given a value for $\gamma$, the trellis is specified by the probabilities of the state transitions $P\left(S_{n} \mid S_{n-1}\right)$ or equivalently by the conditional probabilities $P\left(I_{n} \mid S_{n-1}\right)$. These are the a priori probabilities (APP) of the Markov model and can be calculated and stored as a lookup table.

To find a proper value for $\gamma$, we examine the redundancies defined as $R(\gamma)=2-H\left(I_{n} \mid S_{n-1}\right)$, where $H\left(I_{n} \mid S_{n-1}\right)$ is the average conditional entropy. Table II shows typical redundancies for values of $\gamma=0,1,2,3,4$ at different mobile speeds in the range of our interest. As expected, it is observed that redundancies are higher for larger values of $\gamma$. However, for $\gamma \geq 2$, redundancies do not increase significantly, which means that there is no significant residual redundancy [32] left in the sequence after that depth. Hence, we use $\gamma=3$ in our simulations, which is a small value, and yet it captures most of the redundancies. The resulting trellis, which has 32 states, is used in some of our algorithms.

To implement (14), a proper value of $\mu$ is also required. Considering (15), the value of $\mu$ should be chosen to include the number of feedback symbols that carry some useful information about the codeword. The redundancies are limited to a certain depth, beyond which a larger $\mu$ does not improve the performance. With the experiment, the value is chosen as $\mu=3$.

2) Time Dependency of the Trellis: As discussed in Section II, the quantization process is subject to the framing structure of mode 1 , which is time dependent. In each slot, $I_{n}$ can adopt one of the four values $\{0,1,2,3\}$, which represents a 2-bit value. One of these bits is the information bit, which is transmitted via the feedback channel, and the other bit is specified by the slot number. In other words, given the time slot, $I_{n}$ attains one of two possible values. Therefore, the trellis structure is periodically time dependent. Fig. 5 shows all possible paths on a typical trellis for $\gamma=2$.

As discussed in Appendix B, there are a total of $(\gamma+1) 2^{\gamma}$ possible states. However, some states occur only at the frame boundary, whereas the others can occur within a frame. In other words, in each time slot specified by $\tau$, some states never happen. For $2^{\gamma}$ states (corresponding to $n_{\text {win }}=0,2,4, \cdots, 12$, in Fig. 12), the next symbol could be any of the four possible values because those states can occur both in the middle of a frame and at the boundary. Assuming that the slot number is known, these states can be split into two categories subject to different framing effects. Hence, given the slot number $\tau$, each state is connected to only two states, as can be observed in Fig. 5. Therefore, it is appropriate to use $P\left(S_{n} \mid S_{n-1}, \tau\right)$ instead of $P\left(S_{n} \mid S_{n-1}\right)$ to capture the time dependency of the trellis. Because there are less possible paths on the trellis, the time- based approach can decrease the complexity of the trellis by a factor of $2(\gamma+1)$.

\section{B. Normalized MMSE Algorithm (NMMSE)}

We are dealing with the estimation of a complex variable with a constant amplitude as $\left|\hat{w}_{n}^{(2)}\right|=1 / \sqrt{2}$. However, there is no control on the amplitude of the MMSE estimate $\check{w}_{n}^{(2)}$ provided by (14) (please note the difference between $\hat{w}$ and $\check{w}$ ). Hence, we need an MMSE estimator with a constant amplitude constraint, which is introduced in Lemma 1 (see Appendix A). According to Lemma 1, we can always calculate the needed antenna weight using the MMSE solution of (14) as

$$
\hat{w}_{n}^{(2)}=\frac{1}{\sqrt{2}} \frac{\check{w}_{n}^{(2)}}{\left|\check{w}_{n}^{(2)}\right|} .
$$

Implementing (14), the probabilities of the possible states of the trellis are required at each time. We have assumed a Markov model and a memoryless feedback channel, so using the Bahl, Cocke, Jelinek, and Raviv (BCJR) algorithm [33], the state probabilities can be computed recursively by [31]

$$
\begin{aligned}
P\left(S_{n} \mid \underline{J}_{n}\right)=C \cdot & P\left(J_{n} \mid I_{n}\right) \\
& \cdot \sum_{S_{n-1} \rightarrow S_{n}} P\left(I_{n} \mid S_{n-1}\right) P\left(S_{n-1} \mid \underline{J}_{n-1}\right)
\end{aligned}
$$

where the summation is over all the possible transitions from the states at time $n-1$ to $S_{n}$, and $C$ is the normalizing factor such that $\sum_{S_{n}} P\left(S_{n} \mid \underline{J}_{n}\right)=1 . P\left(I_{n} \mid S_{n-1}\right)$ 's are available as APP, and $P\left(J_{n} \mid I_{n}\right)$ 's are the channel transition probabilities. Assuming a binary symmetric channel (BSC) with error probability of $p_{e}$ for transmitted feedback bits, the equivalent feedback channel (Fig. 2) is represented by $P\left(J_{n} \mid I_{n}\right)$, where $P\left(I_{n} \mid I_{n}\right)=1-p_{e}$, and $P\left(J_{n} \mid I_{n}\right)=p_{e}$ if $J_{n} \neq I_{n}$, but both belong to the same quantization set; otherwise, $P\left(J_{n} \mid I_{n}\right)=0$.

For simplicity, we assume that $\mu \leq \gamma$. Then, the required probabilities of $P\left(\underline{I}_{n}^{n-\mu+1} \mid \underline{J}_{n}\right)$ in (14) are directly calculated using (17) as

$$
P\left(\underline{I}_{n}^{n-\mu+1} \mid \underline{J}_{n}\right)=\sum P\left(S_{n} \mid \underline{J}_{n}\right)
$$

where summation is over all the states $S_{n}$ that include the $\underline{I}_{n}^{n-\mu+1}$ sequence. This assumption eliminates the need for backward recursion of the BCJR algorithm.

\section{Nonlinearly Estimated Weight Algorithm (NLW)}

Simplifying the MMSE algorithm, assume that the feedback stream is error free. In this case, (14) reduces to a codeword selection, and Lemma 1 is used to guarantee weight normalization. Because the codeword is a nonlinear estimator of the weight in comparison with the linear codewords of the standard, we call the algorithm as NLW algorithm. 


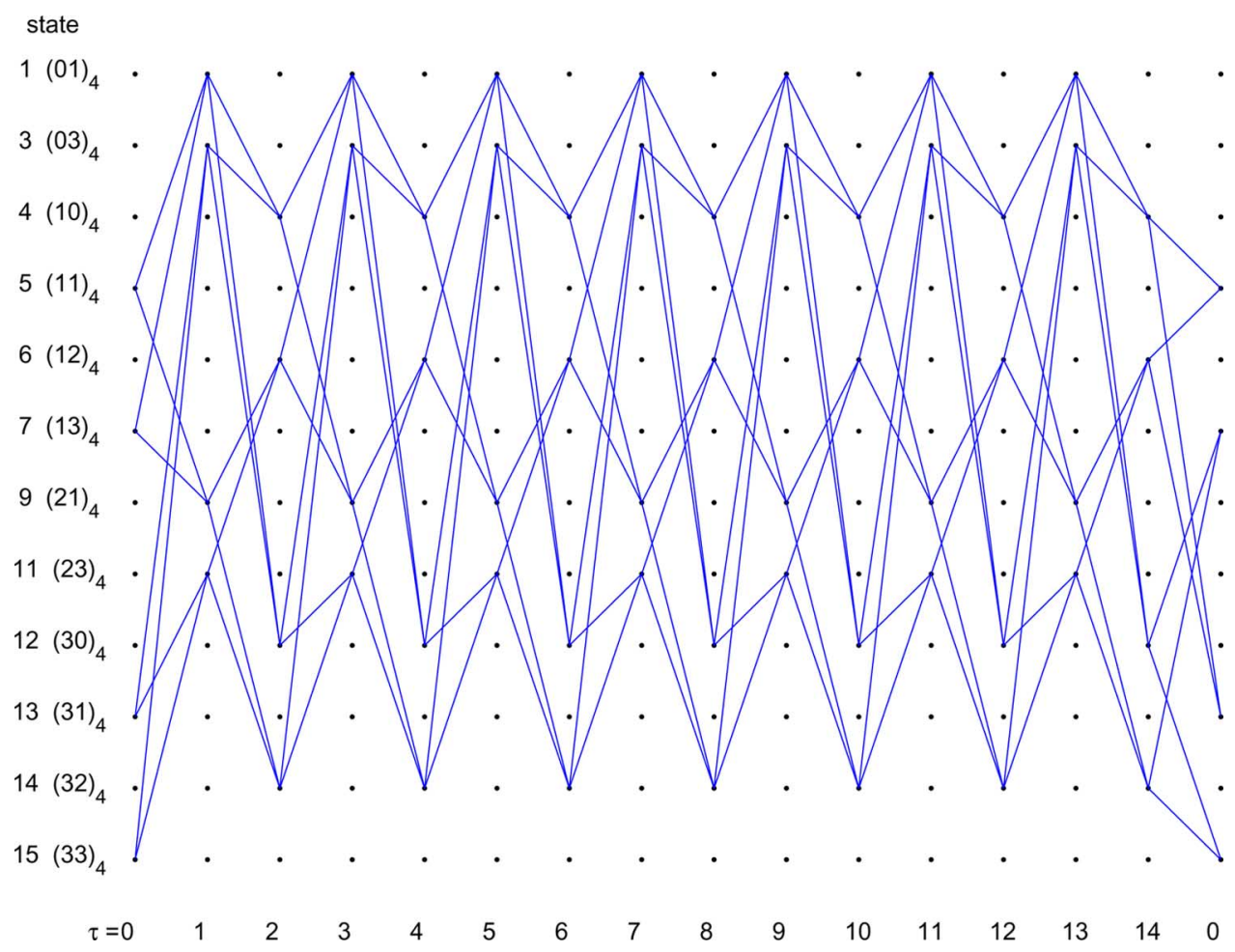

Fig. 5. Time-based trellis for the case $\gamma=2$.

In the NLW algorithm, the received feedback sequence is taken as the estimate of the transmitted sequence $\hat{i}_{n}=j_{n}$, where $j_{n}$ is the hard-decided received feedback symbol, and it is used to generate the index for the codeword, i.e.,

$$
\check{w}_{n}^{(2)}=w_{\mathrm{CB}}\left(\underline{\hat{i}}_{n}^{n-\mu+1}\right)
$$

which is applied to (16). The NLW algorithm is generally suboptimum; however, it is asymptotically optimum for the error-free feedback case.

\section{Sequence MAP Algorithm (SMAP)}

The sequence MAP decoder [31], [34] receives the sequence $\underline{J}_{n}$ and determines the most probable transmitted sequence as $\underline{\underline{i}}_{n}=\arg \max P\left(\underline{I}_{n} \mid \underline{J}_{n}\right)$ using the trellis in Section III-A1. Considering the memoryless property of the feedback channel, the following branch metric can be obtained for the respective trellis [31]:

$$
m\left(J_{n}, S_{n-1} \stackrel{I_{n}}{\rightarrow} S_{n}\right)=\log \left\{P\left(J_{n} \mid I_{n}\right) P\left(I_{n} \mid S_{n-1}\right)\right\}
$$

which is used within the well-known Viterbi algorithm to find the solution. The estimated sequence is applied to (19). Then, the beamforming weight is calculated by (16). Note that the SMAP algorithm could be used to exploit the trellis with a lower computational complexity in comparison with the MMSE solution.
TABLE III

IMPLEMENTATION REQUIREMENTS OF OUR ALGORITHMS AT THE BASE STATION

\begin{tabular}{|c|c|c|c|}
\hline $\begin{array}{c}\text { Our } \\
\text { Algorithm: }\end{array}$ & $\begin{array}{c}\text { NLW } \\
\text { Algorithm }\end{array}$ & $\begin{array}{c}\text { SMAP, } \\
\text { Soft-SMAP }\end{array}$ & $\begin{array}{c}\text { NMMSE, } \\
\text { Soft-NMMSE }\end{array}$ \\
\hline \hline codebook & Yes & Yes & Yes \\
\hline APP & & Yes & Yes \\
\hline Trellis Processing & & Viterbi & Forward-BCJR \\
\hline
\end{tabular}

\section{E. Soft-Output Methods (Soft-SMAP and Soft-NMMSE)}

The use of soft feedback data [35] can potentially improve the performance of the SMAP and NMMSE algorithms. In soft methods, it is assumed that instead of hard-decided bits of feedback data, soft-output (noisy) symbols are available. In other words, instead of a BSC feedback channel, an AWGN channel with binary input is assumed. The noise power is selected to keep the hard-decision feedback error probability the same for the two cases.

\section{F. Implementation and Complexity}

Note that the algorithms that have been proposed here are real time and decide on the needed antenna weight without any delay. Receiving the most recent feedback symbol $J_{n}$, each algorithm calculates $\hat{w}_{n}^{(2)}$ to be applied at the transmitter. At the receiver, the transmitted feedback sequence $\underline{I}_{n}=\underline{i}_{n}$ is available. Hence, to calculate the combining variable $v_{n}$, the receiver calculates its estimate of the weights using the codebooks, as in the error-free case. In other words, the base station uses one of the proposed algorithms to calculate the weights, while the mobile unit just needs to select the $\underline{i}_{n}^{n-\mu+1}$ th 


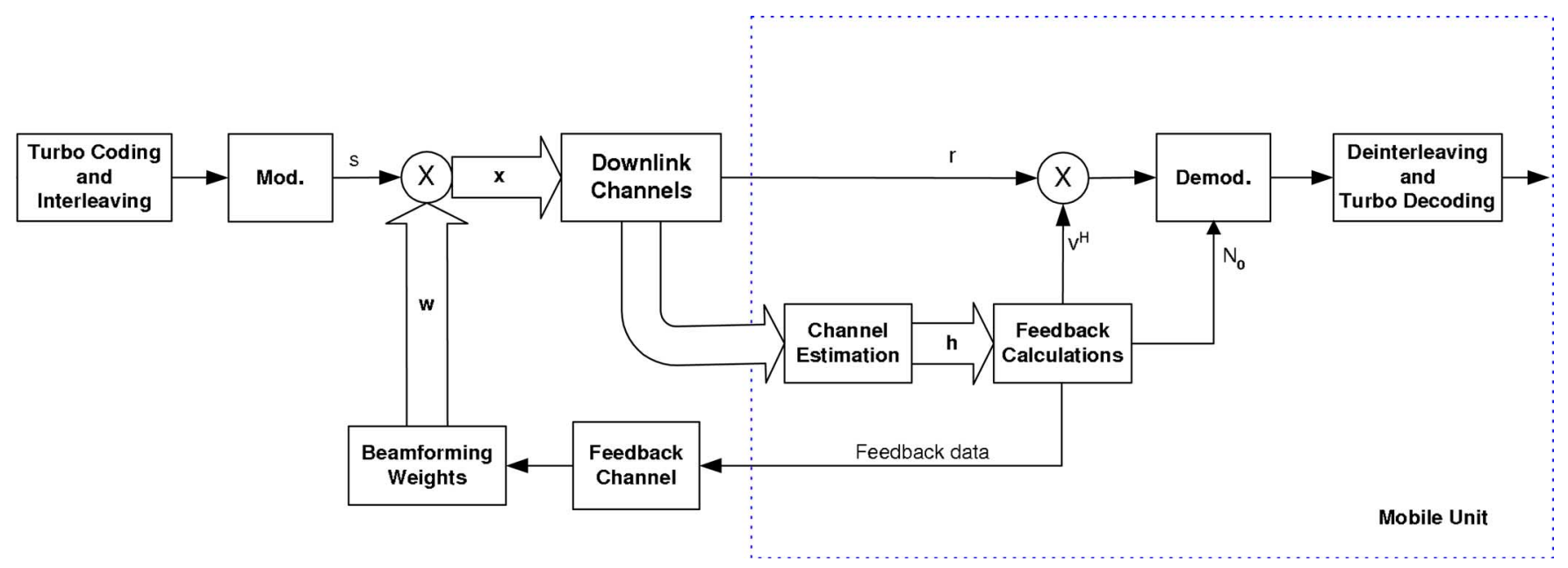

Fig. 6. Block diagram of our feedback system.

weight out of a normalized codebook (similar to the NLW algorithm).

The codebook and APP are calculated offline. The calculation process of the codebooks for different channel models is the same, as explained in Section III-A. We use Jakes' fading model, and these codebooks are calculated once for each mobile speed corresponding to different Doppler frequencies using sufficient amount of training data. For selecting the proper codebook, an estimate of the mobile speed is required. There are various algorithms suggested for a general mobile speed estimation [36] or in particular for 3G systems [37]. It is shown in the simulation results that for the estimation accuracies reported in the above references, the performance of our algorithms does not change significantly. In other words, the proposed algorithms are robust to mobile speed estimation error. Therefore, a small number of codebooks, which correspond to a small number of mobile speeds, are sufficient to cover the entire range.

Regarding the computational complexity, our algorithms use known methods like Viterbi algorithm and forward recursion of the BCJR algorithm with small memory depths. Therefore, the complexity is relatively low. Furthermore, these algorithms are implemented at the base station, where complexity is not of great concern. Although, using the time dependency of the trellis, the complexity of our trellis-based algorithms could be further decreased. Table III shows the requirements of each of our algorithms at the base station. At the mobile unit, only codebooks are required, as the NLW algorithm is implemented regardless of the specific algorithm used at the base station.

\section{RESUlts}

\section{A. Simulation Parameters}

For the testing and comparison of the algorithms, we have simulated a communication system similar to the downlink of FDD WCDMA [4]. Fig. 6 shows our complete system, including the channel coding and the details of the feedback system. As channel code, Turbo coding [39] is used, whose parameters are suggested by the 3GPP standard [1], [40]. In CDMA systems including $3 \mathrm{GPP}$, there is a power control scheme intended
TABLE IV SIMULATION PARAMETERS

\begin{tabular}{|c|c}
\hline Carrier Frequency & $2.15 \mathrm{GHz}$ \\
\hline Modulation & QPSK \\
\hline Transmitter & 2 Antennas \\
\hline Receiver & 1 Antenna \\
\hline Data Rate & $15000 \mathrm{bps}$ \\
\hline Feedback Rate & $1500 \mathrm{bps}$ \\
\hline Channel Model & Modified Jakes \\
\hline \hline Channel Coding & Turbo Code \\
\hline Code Rate & $1 / 3$ \\
\hline Frame Length & $300(20 \mathrm{mSec})$ \\
\hline Bit Interleaving & One Frame \\
\hline \hline Channel Estimation & Ideal \\
\hline Power Control & Not applied \\
\hline
\end{tabular}

to eliminate slow fading. Therefore, we assume that slow fading is compensated by power control. Table IV is a summary of the parameters of our simulations. The other parameters that may change in different simulations are specified in each case. Also, no antenna verification is used with the closed-loop algorithms unless stated otherwise.

\section{B. Simulation Results}

As a measure of performance, we use frame error rate (FER), which is appropriate for a coded communication system. The overall performance of the system is determined by the interplay of feedback error rate and mobile speed. Feedback error degrades the beamforming performance and can result in an error floor effect as mentioned before. On the other hand, increasing the mobile speed improves the decoding performance in the forward link (as a result of increasing the fading diversity) but generally degrades the performance of the feedback reconstruction algorithms to different extents. Therefore, given a feedback error rate, algorithms perform diversely over different mobile speeds.

The following algorithms have been considered in the simulations: Standard (closed-loop mode 1 of 3GPP), NLW, SMAP, Soft-SMAP, NMMSE, and Soft-NMMSE, which are in ascending order of complexity. It is assumed that $\gamma=3$, and $\mu=3$. Fig. 7 shows the FER performance of the algorithms versus transmit SNR for 5\% feedback error and for the mobile speeds 

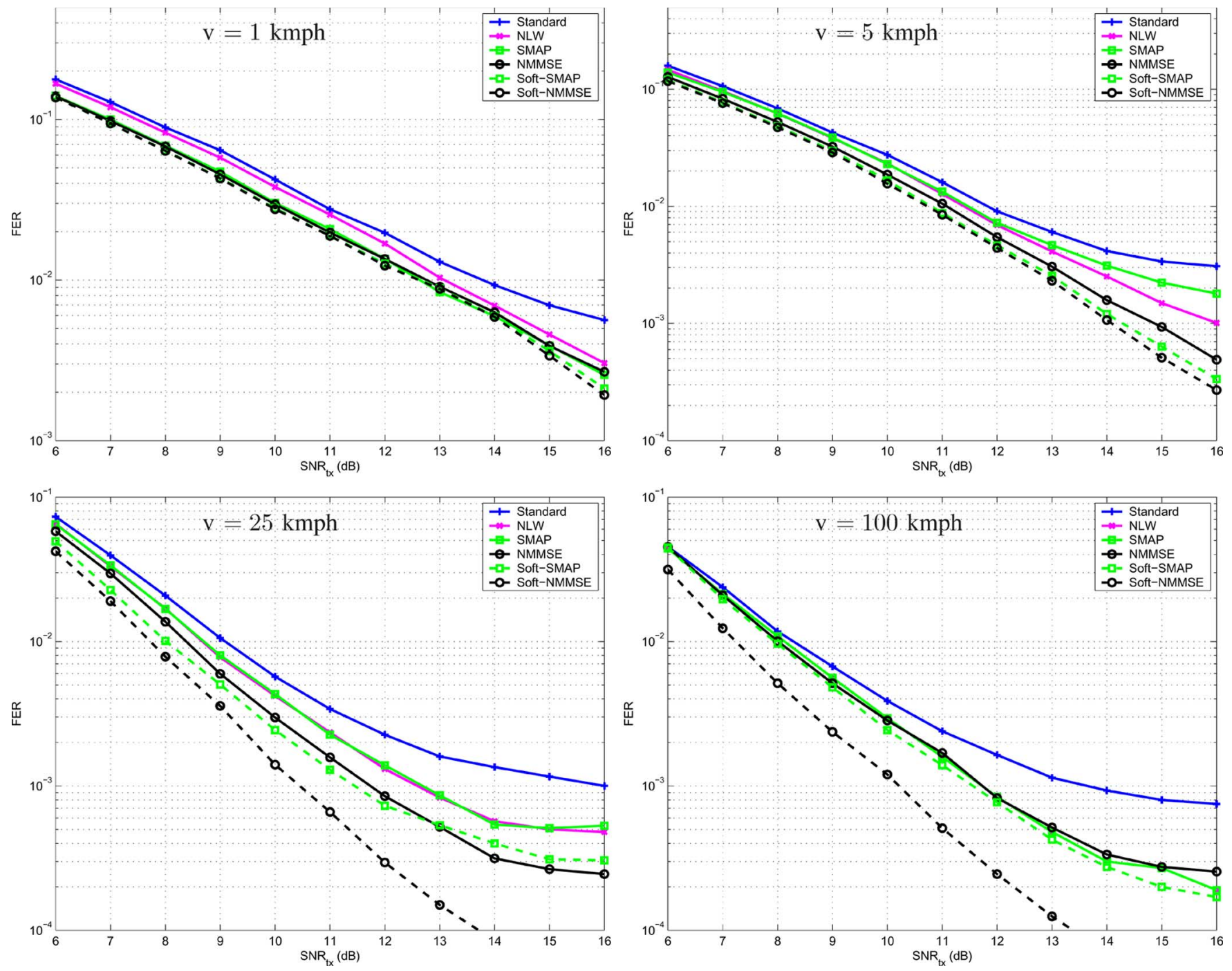

Fig. 7. FER versus transmit SNR in 5\% feedback error.

of $v=1,5,25$, and $100 \mathrm{~km} / \mathrm{h}$. Fig. 8 similarly shows the FER in $10 \%$ feedback error.

It is observed in Figs. 7 and 8 that our algorithms outperform the standard and result in significant savings in transmit power. Regarding the relative gain of the algorithms, Figs. 9 and 10 show the required SNR for a fixed FER versus mobile speed in $5 \%$ and $10 \%$ of feedback error, respectively. The plots include the standard algorithm with an ideal $\mathrm{AV}$, as explained in Section II-B. The performance of the Standard-ideal antenna verification (IAV) is the best performance that is achievable by using an AV algorithm along with the standard algorithm; hence, it is a performance bound on using an $\mathrm{AV}$ algorithm. It is observed that NMMSE and Soft-NMMSE perform similar or even better than the Standard-IAV bound for low to moderate mobile speeds. Fig. 9 shows that our approach can decrease the transmit power by more than $2 \mathrm{~dB}$ for low to high mobile speeds. This gain is higher for a smaller target FER or for a higher feedback error rate.

Regarding the effect of mobile speed and feedback error rate on performance, the following observations can be made from Figs. 7-10: at low mobile speeds, the reconstruction algorithms perform in this ascending order: first Standard, then NLW, and then the rest of the algorithms (which utilize APP) perform similarly, in particular at low SNRs. With increasing speed, the performance of the algorithms starts to differ. Soft-NMMSE and NMMSE algorithms outperform their SMAP counterparts. As expected, utilizing soft output improves the performance of the corresponding algorithms. At high mobile speeds, the channel changes rapidly, and the redundancy in the feedback bit stream diminishes. Therefore, the SMAP approach reduces to maximum likelihood detection [see (20)], and Soft-SMAP performs like SMAP. (This phenomenon is similarly observed in [31, Fig. 9]). However, the Soft-NMMSE algorithm still efficiently utilizes the soft outputs and substantially outperforms the other algorithms. Note that in each algorithm, the effect of error floor on FER is higher for a larger feedback error rate and at higher SNRs. The Soft-NMMSE algorithm provides the best performance at all mobile speeds and feedback error rates.

1) Sensitivity to Speed Estimation Error: As explained before, codebook and APP are chosen using an estimate of the mobile speed. Hence, the effect of estimation error on 

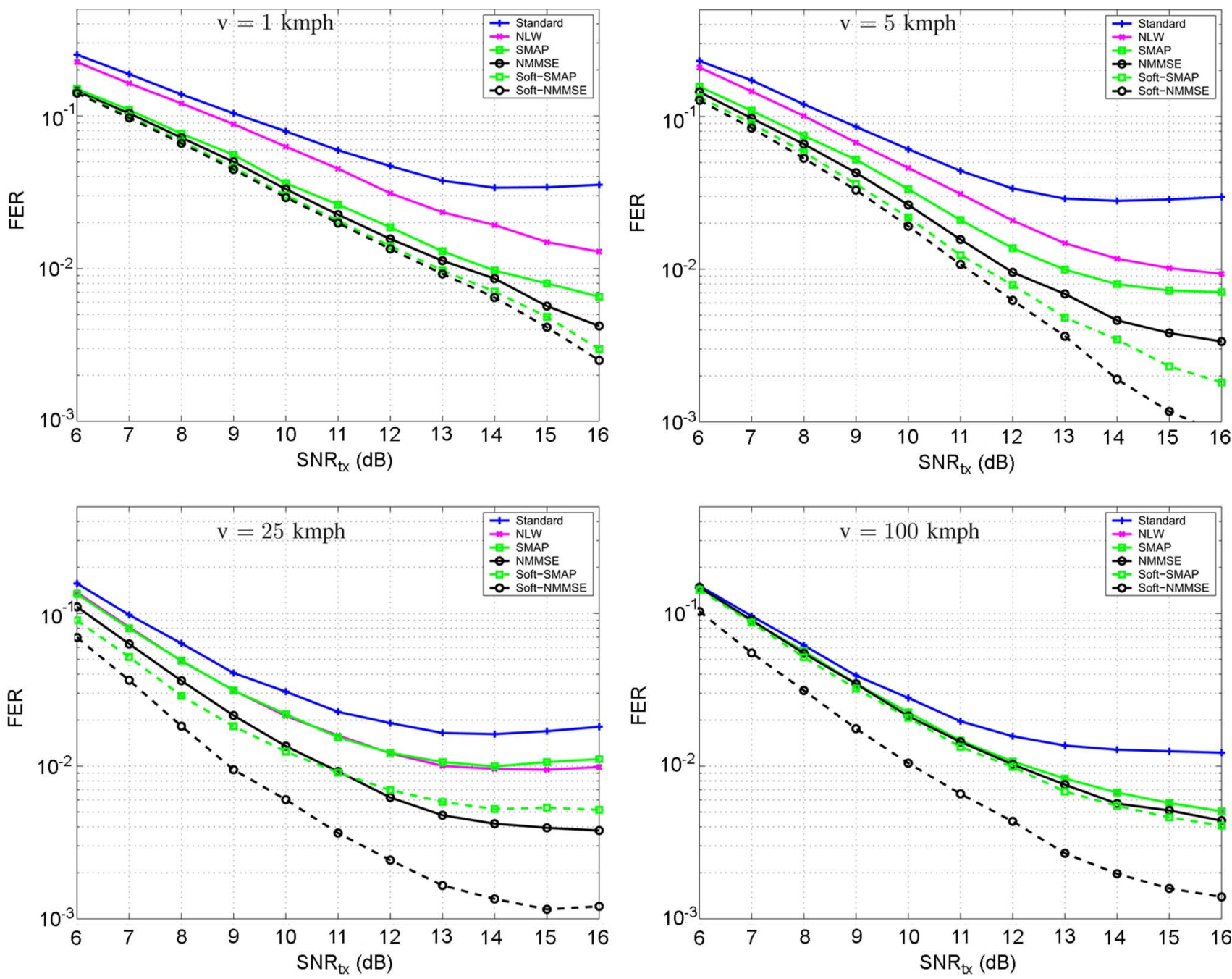

Fig. 8. FER versus transmit SNR in $10 \%$ feedback error

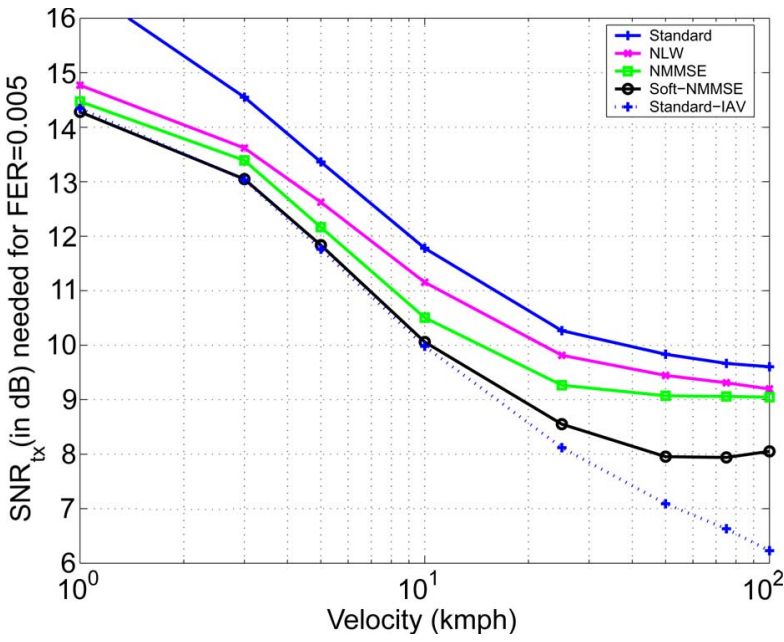

Fig. 9. Required SNR for FER $=5 \mathrm{e}-3$ in $5 \%$ feedback error.

performance is investigated. As an example, we consider the Soft-NMMSE algorithm, which has the best performance among our algorithms and uses both codebook and APP. At a given mobile speed, we apply the codebooks related to a higher and a lower mobile speed to the algorithm, as shown

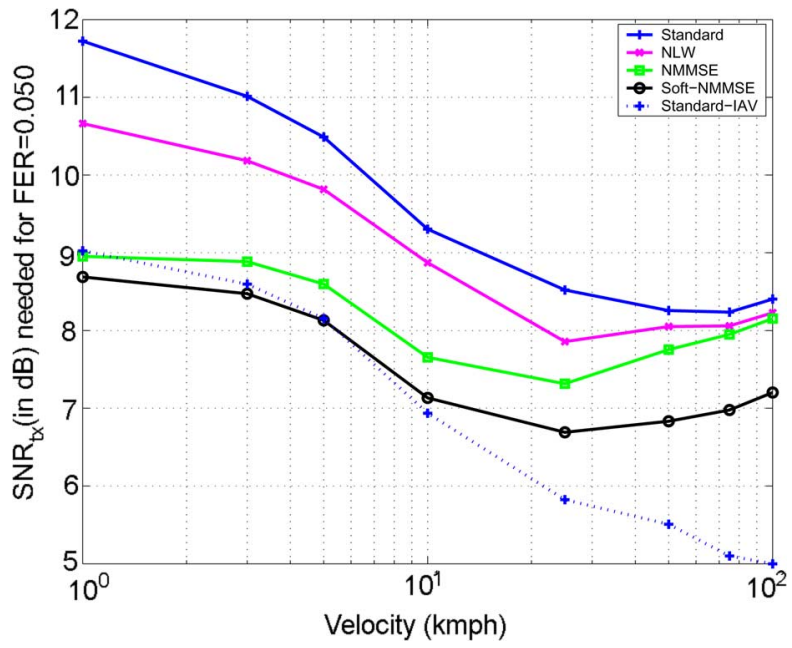

Fig. 10. Required SNR for FER $=5 \mathrm{e}-2$ in $10 \%$ feedback error.

in Table V. The estimation errors used in the table are higher than the ranges reported for speed estimation algorithms [36]-[38]. The results shown in Fig. 11 indicate that the algorithm is not sensitive to the mobile speed estimation error. 
TABLE V

Actual, Lower, And Higher Estimates of the Mobile SpeEd

\begin{tabular}{|c|c|c|c|c|c|c|c|}
\hline Actual Speed (kmph) & 3 & 5 & 10 & 25 & 50 & 75 & 100 \\
\hline Lower Estimate & 1 & 1 & 5 & 15 & 40 & 60 & 80 \\
\hline Higher Estimate & 5 & 10 & 15 & 30 & 60 & 90 & 125 \\
\hline
\end{tabular}

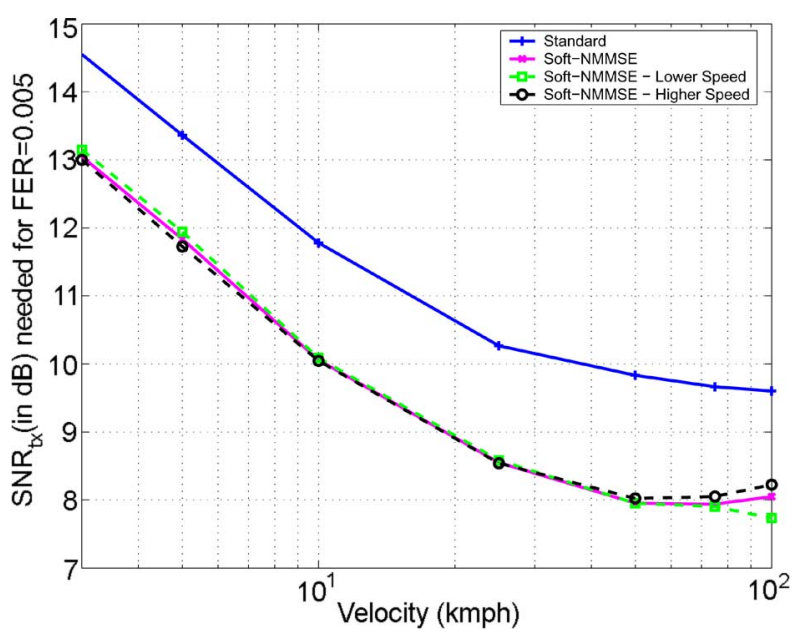

Fig. 11. Required SNR for FER $=5 \mathrm{e}-3$ in $5 \%$ feedback error: sensitivity to the speed estimation error.

Therefore, a limited number of codebooks can be used to cover the entire range of mobile speeds.

\section{Concluding Remarks}

The closed-loop transmit diversity, which uses a combination of transmit diversity and channel feedback, is recognized as a promising approach to achieve high data rates in mobile communications. In 3GPP systems, however, the performance of the closed-loop scheme is limited, which is the result of the rate limit and error in the feedback channel and also the nonoptimum weight reconstruction algorithm suggested in the standard. In this paper, we propose an approach to improve the performance of the closed-loop system in the presence of feedback error without changing the structure of the standard. We have introduced a number of algorithms to improve the performance of mode 1 of $3 \mathrm{GPP}$. It has been shown that our algorithms can provide significant gains over the conventional approach at all mobile speeds. The performance of our algorithms, which do not need any preamble, is usually good enough such that an $\mathrm{AV}$ algorithm is no longer required. Although, an AV could be used along with our algorithms for further improvement. Moreover, our proposed algorithms are implemented mostly at the base station, as opposed to AV, which increases the complexity of the mobile unit.

In this paper, we have tested the proposed algorithms with Jakes' fading. A thorough examination of the performance at different scattering environments can be the subject of a continuation of this paper.

\section{APPENDIX A LEMMA 1}

Lemma 1: Consider the random variable $w$ that $|w|=\beta$. Assume that $\hat{w}$ is the constant-amplitude MMSE estimation of $w$. Given $|\hat{w}|=\alpha$, then

$$
\hat{w}=\alpha \frac{E[w]}{|E[w]|} .
$$

Proof: $\hat{w}=\alpha e^{j \hat{\phi}}$ should be estimated using the random variable $w=\beta e^{j \phi}$, where $\alpha$ and $\beta$ are real positive constants. In the MMSE sense, it turns out to minimizing the following criterion:

$$
\begin{aligned}
& E\left[|w-\hat{w}|^{2}\right] \\
= & E\left[\left|\beta e^{j \phi}-\alpha e^{j \hat{\phi}}\right|^{2}\right] \\
= & \alpha^{2}+\beta^{2}-2 \alpha \beta E[\cos (\phi-\hat{\phi})]
\end{aligned}
$$

or maximizing

$$
\begin{aligned}
& E[\cos (\phi-\hat{\phi})] \\
= & E[\cos \phi \cos \hat{\phi}+\sin \phi \sin \hat{\phi}] \\
= & E[\cos \phi] \cos \hat{\phi}+E[\sin \phi] \sin \hat{\phi}
\end{aligned}
$$

which results in

$$
\tan \hat{\phi}=\frac{E[\sin \phi]}{E[\cos \phi]}
$$

where

$$
\left\{\begin{array}{l}
E[\cos \phi] \geq 0 \Rightarrow-\frac{\pi}{2}<\hat{\phi} \leq \frac{\pi}{2} \\
E[\cos \phi]<0 \Rightarrow \frac{\pi}{2}<\hat{\phi} \leq 3 \frac{\pi}{2}
\end{array}\right.
$$

Finding the phase information of $\hat{w}$, i.e., $e^{j \hat{\phi}}=\cos \hat{\phi}+j \sin \hat{\phi}$, from (28), it is easy to show that in both cases of (29)

$$
e^{j \hat{\phi}}=\frac{E\left[e^{j \phi}\right]}{\left|E\left[e^{j \phi}\right]\right|} .
$$

Therefore

$$
\hat{w}=\alpha e^{j \hat{\phi}}=\alpha \frac{E\left[e^{j \phi}\right]}{\left|E\left[e^{j \phi}\right]\right|}=\alpha \frac{E[w]}{|E[w]|} .
$$

\section{APPENDIX B \\ Calculation of the Number of States (OR THE CODEBOOK SIZE)}

Consider the framing structure of mode 1 of $3 \mathrm{GPP}$, as depicted in Fig. 12. In the figure, each slot shows the quantizer used according to Section II. At time slot $n$, the current state is composed of $\gamma$ symbols, from the starting slot number $n_{\text {win }}=n-\gamma+1$ to the slot number $n$, which is shown by dashed sliding windows. The windows shown in the figure correspond to the case $\gamma=3$, but our discussion is for any value of $0<\gamma<15$. For a given window specified by $n_{\text {win }}$, there are 


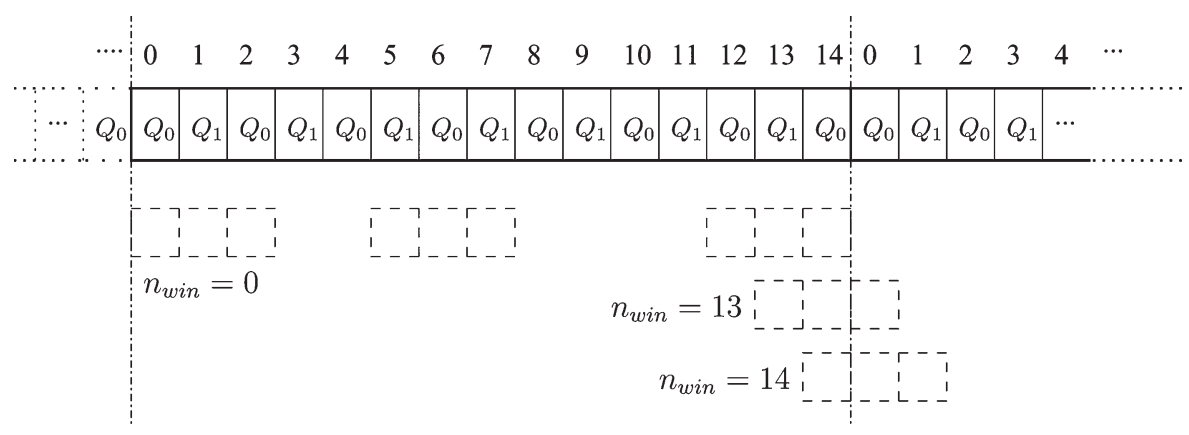

Fig. 12. Calculation of the number of states.

$2^{\gamma}$ possible states. Depending on the window position, there are two cases:

Case 1: For $n_{\text {win }}=0,1, \ldots, 15-\gamma$, the window is within one frame, and the symbols are consecutively selected from $Q_{0}$ and $Q_{1}$. Therefore, for all of the odd $n_{\text {win }}$ 's, the sequence of possible subsets is the same. This is also the case for all even $n_{\text {win }}$ 's. Hence, there are two possible sequences of subsets for this range of $n_{\text {win }}$, which results in $2 \times 2^{\gamma}$ different states.

Case 2: For $n_{\text {win }}=16-\gamma, \ldots, 14$, the window is in two neighboring frames. The symbols are also consecutively selected from $Q_{0}$ and $Q_{1}$ except for the slots at the boundary of the two frames where symbols are selected from two consecutive $Q_{0}$ 's. Therefore, for each $n_{\text {win }}$ in the range, the sequences of possible subsets are unique and different from Case 1. Hence, there are $14-(16-\gamma)+1=\gamma-1$ possible sequences of subsets for this range of $n_{\text {win }}$, which results in $(\gamma-1) 2^{\gamma}$ different states.

Adding up the possible states of the two cases, it leads to $(\gamma+1) 2^{\gamma}$ possible states for the structure of mode 1 of 3GPP. Note that a similar deduction could be done to calculate the codebook size by replacing $\gamma$ with $\mu$, which results in $(\mu+1) 2^{\mu}$.

\section{REFERENCES}

[1] A. Heidari, F. Lahouti, and A. K. Khandani, "Improved reconstruction of channel state information in 3GPP," in Proc. VTC - Fall, Dallas, TX, Sep. 2005, pp. 1998-2002.

[2] The 3GPP Website. [Online]. Available: http://www.3gpp.org

[3] The 3GPP2 Website. [Online]. Available: http://www.3gpp2.org

[4] "3GPP technical specification," UMTS Physical Layer: General Description, Dec. 2003. ETSI TS 125201 V6.0.0.

[5] I. E. Teletar, "Capacity of multi-antenna Gaussian channels," in "Technical Memorandum," Bell Lab., Lucent Technol., Murray Hill, NJ, Oct. 1995.

[6] R. T. Derryberry et al., "Transmit diversity in 3G CDMA systems," IEEE Commun. Mag., vol. 40, no. 4, pp. 68-75, Apr. 2002.

[7] V. Tarokh, N. Seshadri, and A. R. Calderbank, "Spacetime codes for high data rate wireless communication: Performance criterion and code construction," IEEE Trans. Inf. Theory, vol. 44, no. 2, pp. 744-765, Mar. 1998

[8] S. M. Alamouti, "A simple transmitter diversity scheme for wireless communications," IEEE J. Sel. Areas Commun., vol. 16, no. 8, pp. 1451-1458, Oct. 1998.

[9] "3GPP technical specification," UMTS Physical Layer Procedures (FDD), Dec. 2003. ETSI TS 125214 V6.0.0.

[10] E. Visotsky and U. Madhow, "Space-time precoding with imperfect feedback," IEEE Trans. Inf. Theory, vol. 47, no. 6, pp. 2632-2639, Sep. 2001.

[11] H. Gerlach, "SNR loss due to feedback quantization and errors in closed loop transmit diversity systems," in Proc. 13th IEEE Int. Symp. PIMRC, Sep. 2002, pp. 2117-2120.
[12] J. Hamalainen and R. Wichman, "Performance analysis of closed-loop transmit diversity in the presence of feedback errors," in Proc. Int. Symp. Pers., Indoor, Mobile Radio Commun., Sep. 2002, pp. 2297-2301.

[13] J. Hamalainen and R. Wichman, "The effect of feedback delay to the closed-loop transmit diversity in FDD WCDMA," in Proc. Int. Symp. Pers., Indoor, Mobile Radio Commun., Oct. 2001, pp. D-27-D-31.

[14] A. Hottinen and R. Wichman, "Transmit diversity using filtered feedback weights in the FDD/WCDMA system," in Proc. Broadband Commun., Feb. 2000, pp. 15-21.

[15] J. G. Proakis, Digital Communications. New York: McGraw-Hill, 2001. Mc-Graw Hill International Editions.

[16] M. F. Pop and N. C. Beauliu, "Limitations of sum-of-sinusoids fading channel simulators," IEEE Trans. Commun., vol. 49, no. 4, pp. 699-708, Apr. 2001.

[17] W. C. Jakes, Ed., Microwave Mobile Communications. New York: IEEE Press, 1974.

[18] A. Seeger and M. Sikora, "Antenna weight verification for closedloop transmit diversity," in Proc. IEEE GLOBECOM Conf., Dec. 2003 pp. $1124-1129$.

[19] A. Narula, M. J. Lopez, M. D. Trott, and G. W. Wornell, "Efficient use of side information in multiple-antenna data transmission over fading channels," IEEE J. Sel. Areas Commun., vol. 16, no. 8, pp. 1423-1436, Oct. 1998.

[20] B. A. Bjerke, Z. Zvonar, and J. G. Proakis, "Antenna diversity combining schemes for WCDMA systems in fading multipath channels," IEEE Trans. Wireless Commun., vol. 3, no. 1, pp. 97-106, Jan. 2004.

[21] P. A. Dighe, R. K. Mallik, and S. S. Jamuar, "Analysis of transmit-receive diversity in Rayleigh fading," IEEE Trans. Commun., vol. 51, no. 4, pp. 694-703, Apr. 2003.

[22] A. Narula, M. D. Trott, and G. W. Wornell, "Performance limits of coded diversity methods for transmitter antenna array," IEEE Trans. Inf. Theory, vol. 45, no. 7, pp. 2418-2433, Nov. 1999.

[23] D. J. Love, R. W. Heath, and T. Strohmer, "Grassmannian beamforming for multiple-input multiple-output wireless systems," IEEE Trans. Inf. Theory, vol. 49, no. 10, pp. 2735-2747, Oct. 2003.

[24] K. C. Hwang and K. B. Lee, "Efficient weight vector representation for closed-loop transmit diversity," IEEE Trans. Commun., vol. 52, no. 1, pp. 9-16, Jan. 2004.

[25] K. K. Mukkavilli, A. Sabharwal, E. Erkip, and B. Aazhang, "On beamforming with finite rate feedback in multiple-antenna systems," IEEE Trans. Inf. Theory, vol. 49, no. 10, pp. 2562-2579, Oct. 2003.

[26] M. Edlund, M. Skoglund, and B. D. Rao, "On the performance of closedloop transmit diversity with non-ideal feedback," in Proc. IEEE ICC, May 2003, pp. 3190-3194.

[27] Texas Instruments, Modified Closed Loop Modes for WCDMA, Aug. 1999. 3GPP TSGR1 7(99)c86.

[28] A. Seeger, M. Sikora, and W. Utschick, "Antenna weight verification for closed-loop downlink eigenbeamforming," in Proc. IEEE GLOBECOM Conf., Nov. 2002, pp. 982-986.

[29] A. Serratore and E. Messina, "Analytical evaluation and performance analysis of antenna verification algorithm in closed loop antenna diversity," in Proc. 9th APCC, Sep. 2003, pp. 9-14.

[30] B. Raghothaman, R. T. Derryberry, and G. Mandyam, "Transmit adaptive array without user-specific pilot for 3G CDMA," in Proc. IEEE ICASSP, Jun. 2000, pp. 3009-3012.

[31] F. Lahouti and A. K. Khandani, "Efficient source decoding over memoryless noisy channels using higher order Markov models," IEEE Trans. Inf. Theory, vol. 50, no. 9, pp. 2103-2118, Sep. 2004. 
[32] K. Sayood and J. C. Brokenhagen, "Use of residual redundancy in the design of joint source-channel coders," IEEE Trans. Commun., vol. 39, no. 6, pp. 838-846, Jun. 1991.

[33] L. Bahl, J. Cocke, F. Jelinek, and J. Raviv, "Optimal decoding of linear codes for minimizing symbol error rate," IEEE Trans. Inf. Theory, vol. IT-20, no. 2, pp. 284-287, Mar. 1974.

[34] S. Emami and S. L. Miller, "DPCM picture transmission over noisy channels with the aid of a Markov model," IEEE Trans. Image Process., vol. 4, no. 11, pp. 1473-1481, Nov. 1995.

[35] N. Phamdo and F. Alajaji, "Soft-decision demodulation design for COVQ over white, colored, and ISI Gaussian channels," IEEE Trans. Commun., vol. 48, no. 9, pp. 1499-1506, Sep. 2000.

[36] L. Zhao and J. W. Mark, "Mobile speed estimation based on average fade slope duration," IEEE Trans. Commun., vol. 52, no. 12, pp. 2066-2069, Dec. 2004

[37] C. Juncker, P. Toft, and N. Morch, "Speed estimation for WCDMA based on the channel envelope derivative," in Proc. IEEE Workshop SPAWC, Jun. 2003, pp. 527-531.

[38] M. Kirsch and F. Berens, "Mobile speed estimation for 3G mobile radio systems using the normalized autocovariance function," in Proc. Int. Zurich Semin. Broadband Commun., Feb. 2002, pp. 48-1-48-4.

[39] C. Berrou and A. Glavieux, "Near optimum error correcting coding and decoding: Turbo-codes," IEEE Trans. Commun., vol. 44, no. 10, pp. 1261-1271, Oct. 1996.

[40] "3GPP technical specification," UMTS Multiplexing and Channel Coding (FDD), Dec. 2003. ETSI TS 125212 V6.0.0.

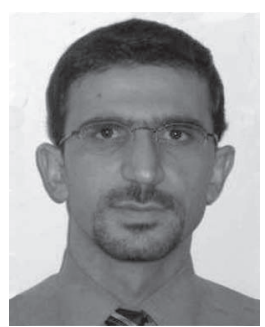

Abdorreza Heidari (S'98) received the B.Sc. and M.Sc. degrees in electronics and telecommunication systems from Sharif University of Technology, Tehran, Iran, in 1997 and 1999, respectively. He is currently working toward the Ph.D. degree in the Department of Electrical and Computer Engineering, University of Waterloo, Waterloo, ON, Canada.

He was a Research Engineer and Project Manager in telecommunication systems and signal processing with Basamad-Negar Company, Tehran. His current research interests are tailored to wireless systems including closed-loop MIMO systems (especially in the presence of feedback imperfections), and modeling and prediction of fading channels.

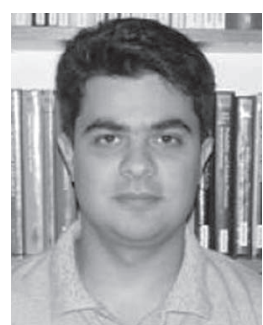

Farshad Lahouti (S'96-A'96-M'02) received the B.Sc. degree in electrical and computer engineering from the University of Tehran, Tehran, Iran, in 1997 and the Ph.D. degree in electrical and computer engineering from the University of Waterloo, Waterloo, ON, Canada, in 2002.

From 2002 to 2004, he was a Postdoctoral Fellow and subsequently a Research Assistant Professor with the University of Waterloo. He is currently with the School of Electrical and Computer Engineering, University of Tehran. His research interests include source/channel coding for multimedia wireless communication networks and DSP applications in digital communications.

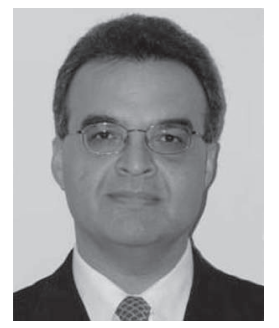

Amir K. Khandani (S'93-M'02) received the M.A.Sc. degree from the University of Tehran, Tehran, Iran, in 1985 and the Ph.D. degree from McGill University, Montreal, QC, Canada, in 1992.

He was a Research Associate with INRS Telecommunications, Quebec University, Montreal, for one year. Since 1993, he has been with the Department of Electrical and Computer Engineering, University of Waterloo, Waterloo, ON, Canada, where he is currently a Professor.

Dr. Khandani is currently holding an NSERC Industrial Research Chair (funded by NSERC and Nortel Networks) on "Advanced Telecommunications Technologies" and a Canada Research Chair (Tier I) on "Wireless Systems." He is serving as an Associate Editor for the IEEE TRANSACTIONS ON COMMUNICATIONS in the area of coding and communication theory. 